\title{
O Teorema Japonês para polígonos cíclicos convexos
}

\author{
Italo Francisconi Vieira JúnioriD
}

Fábio Silva de Souza(D)

\section{Resumo}

O objetivo deste artigo é divulgar um resultado conhecido atualmente como "Teorema Japonês para polígonos cíclicos convexos". Tal teorema foi inscrito em um sangaku japonês durante a Era Edo e afirma que a soma dos inraios dos triângulos gerados pela triangularização de um polígono cíclico convexo, tomada a partir de um dos seus vértices, independe do vértice escolhido. Iremos apresentar a mais antiga prova registrada para quadriláteros e duas provas para polígonos quaisquer. A seguir, mostraremos duas propriedades para quadriláteros, as quais decorrem diretamente do referido teorema, e também uma aplicação prática para o caso geral.

Palavras-chave: teorema japonês; polígonos cíclicos; sangaku.

\begin{abstract}
The purpose of this article is to disclose a result currently known as the "Japanese Theorem for Convex Cyclic Polygons". Such theorem was inscribed in a Japanese sangaku during the Edo Era and states that the sum of the radius of the triangles generated by the triangularization of a convex cyclic polygon, taken from one of its vertices, is independent of the chosen vertex. We will present the oldest registered evidence for quadrilaterals and two evidence for any polygons. Next, we will show two properties for quadrilaterals, which follow directly from the referred theorem, and also a practical application for the general case.
\end{abstract}

Keywords: japanese theorem; cyclic polygons; sangaku.

\section{Introdução}

Conforme escrito em um de seus artigos [1], o professor Mangho Ahuja, da Universidade de Missouri, EUA, encontrou o Teorema Japonês pela primeira vez em 1993, no artigo [13]. Nesse artigo, o autor escreveu "... eu usei o teorema acima como início para um trabalho em andamento, não esperando uma prova do mesmo (eu mesmo não posso provar sozinho)...". A frase estimulou o professor Ahuja a designar tal teorema como problema para Cathy Hawn, um de seus alunos de mestrado. Juntos, Ahuja e Hawn conseguiram prová-lo, e isso pode ser conferido em [9].

Um outro fato que intrigou Ahuja foi o nome do teorema. Por que Teorema Japonês? Após algum tempo de espera, o professor H.Yoshida, da Universidade de Kyoto, enviou a tão esperada resposta. O teorema, caso quadrilátero, teve origem na China e acabou indo parar no Japão, onde ficou conhecido como Teorema Chinês [10]. Mesmo quando Yoshio Mikami [14] provou sua validade 
para um polígono cíclico convexo, em 1905, o nome permaneceu como Teorema Chinês. Acreditase que por esse teorema ter aparecido sem nome em um artigo intitulado Matemáticas Japonesas [7], em 1906, tenha feito com que os autores seguintes o chamassem de Teorema Japonês.

O Teorema Japonês para quadriláteros foi inscrito em 1800, em uma tábua de madeira em um santuário xintoísta, por Ryõkwan Maruyama. De acordo com Fukagawa [6], a procura por tábuas com teoremas matemáticos, chamadas sangaku, era um costume do povo japonês no período conhecido como Era Edo ou Era Tokugawa (1603 - 1868). Exibir em santuários tábuas contendo problemas matemáticos, com figuras elegantes e coloridas, geralmente tinha como objetivo honrar os autores, agradecer aos deuses pelo feito atingido ou desafiar novos visitantes.

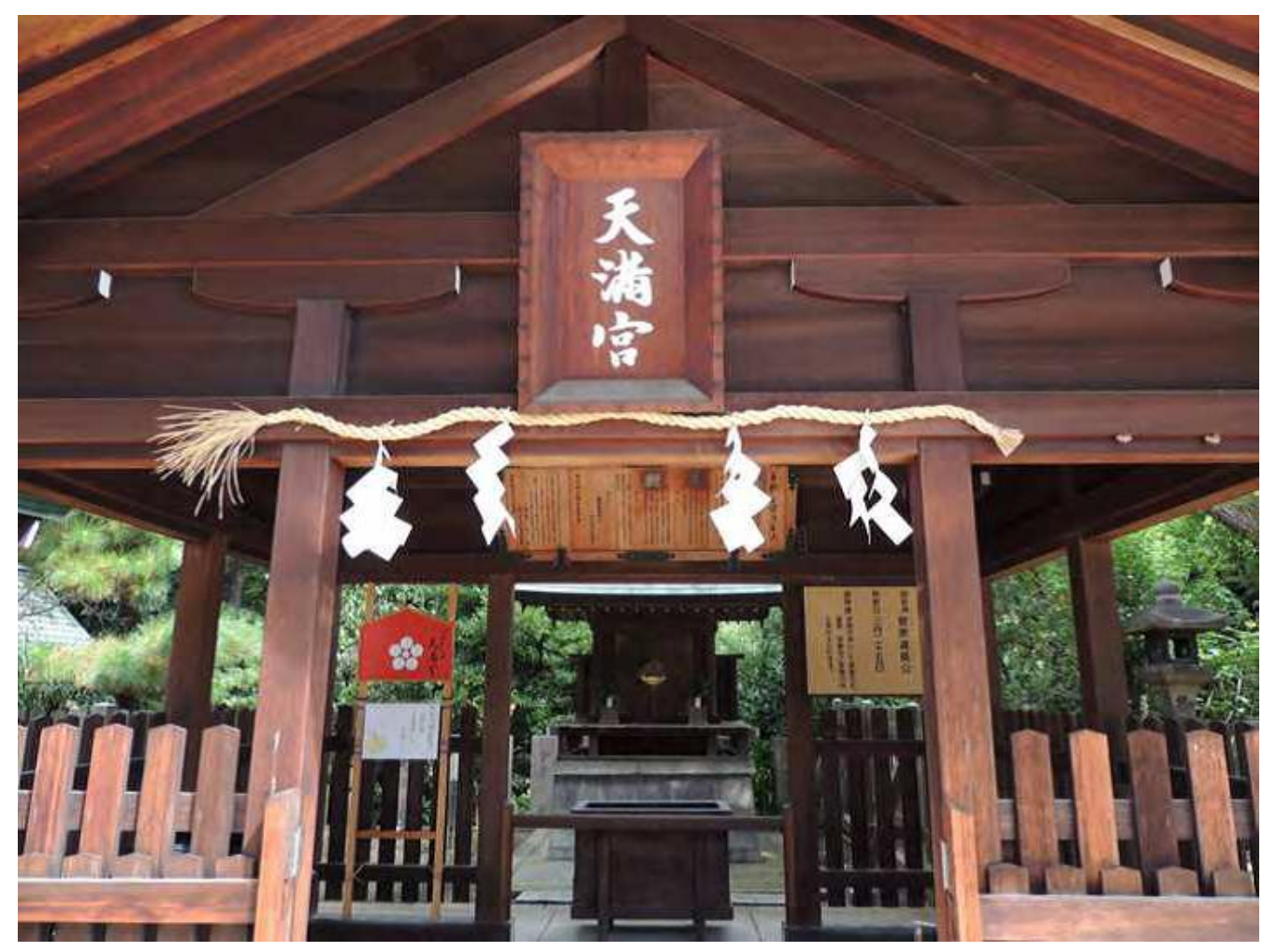

Figura 1: Sangaku exposto no subsantuário Tenman-gū do santuário de Ikutama, Osaka. Fonte: [11], p. 29.

A Figura 1 mostra-nos um exemplo de como os sangaku eram expostos em templos japoneses. Nas Figuras 2 e 3 podemos ver dois sangaku, que são traduzidos e resolvidos em [11]. Já a Figura 4 mostra-nos uma conferência sobre matemática japonesa realizada no Randolph-Macon College, em 2017, nos Estados Unidos. 


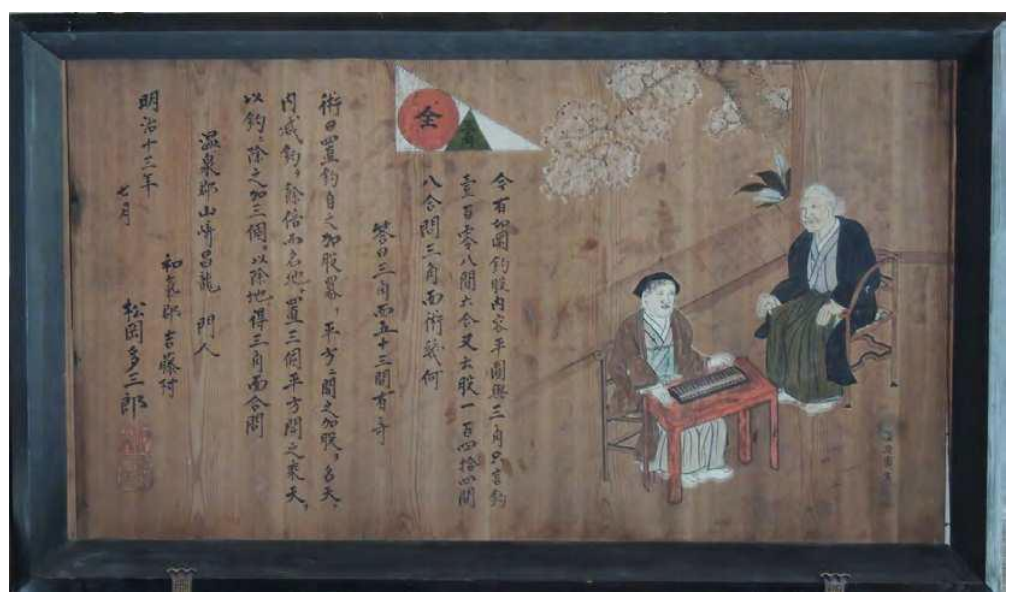

Figura 2: Sangaku do santuário Yoshifuji Mishima, prefeitura de Ehime. Fonte: [11], p. 69.

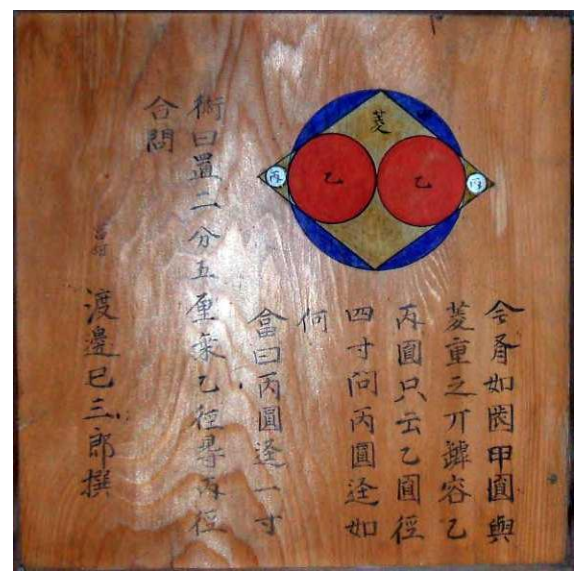

Figura 3: Sangaku do santuário Miharu Itsukushima, prefeitura de Fukushima. Fonte: [11], p. 170. 


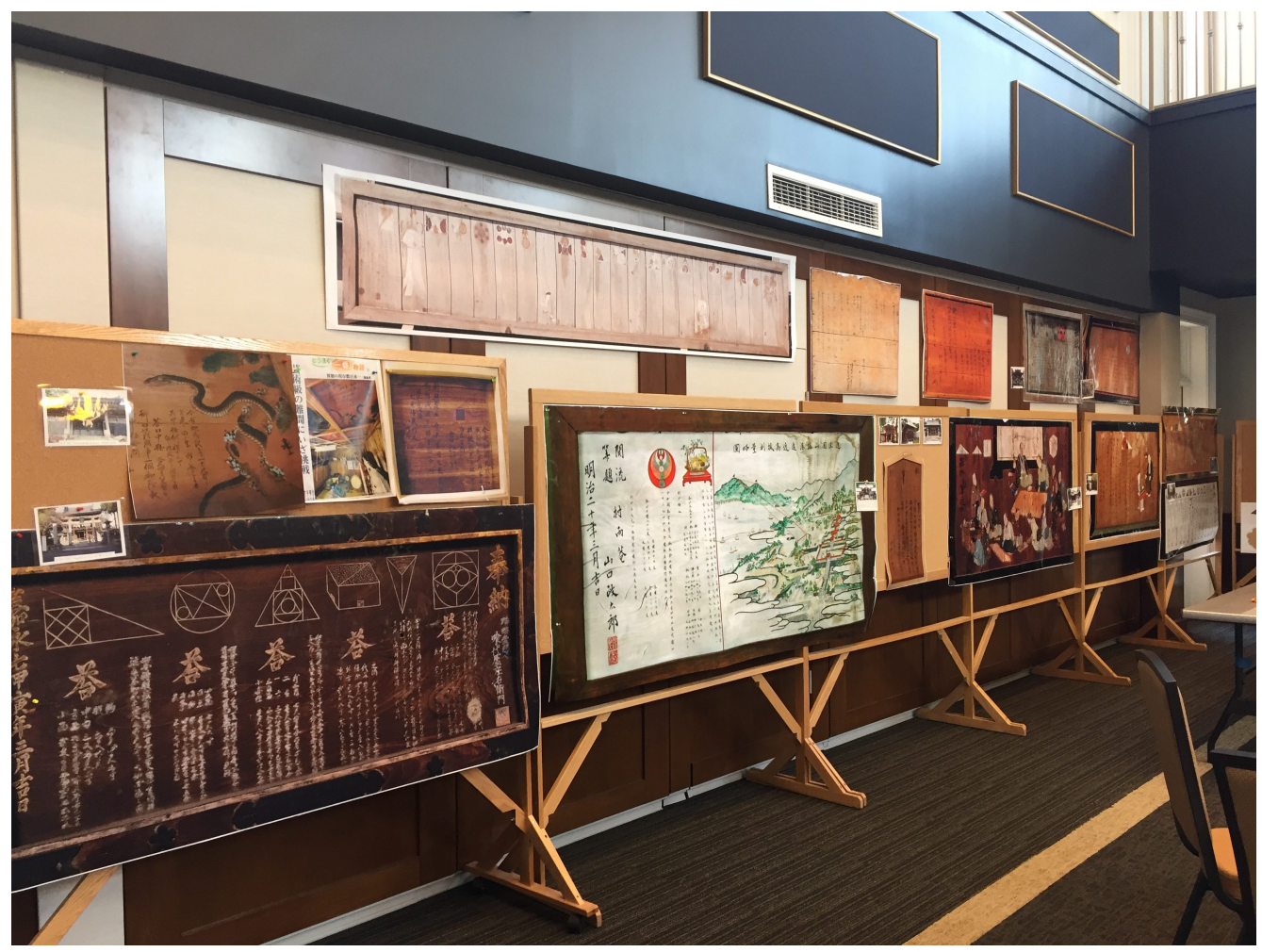

Figura 4: Conferência sobre sangaku e wasan realizada no Randolph-Macon College, 2017. Fonte: <https://folios.rmc.edu/swarm/photographs/>. Acesso em: 26 de setembro de 2021.

O sangaku do Teorema Japonês para quadriláteros pertenceu ao santuário Tsuruoka - San'nosha, na área Ushu, onde atualmente se localizam os municípios de Yamagata e Akita. Apesar de ter desaparecido, sua inscrição foi registrada no segundo volume do Zoku-Sinpeki-Sanpõ de Kagen Fujita [5], em 1807. Conforme tradição, Maruyama também incluiu a resposta e uma breve descrição de como chegar a ela, chamada "arte do problema". Porém, ele não incluiu nenhuma prova. Segundo Ahuja, Uegaki e Matsushita [2], a inscrição original dizia:

Desenhe seis linhas no círculo e faça quatro círculos inscritos em três das linhas. Se o diâmetro do círculo sul, leste e oeste é de 1 sol, 2 sóis e 3 sóis, respectivamente, qual o comprimento do diâmetro do círculo norte? Resposta: 4 sóis. Arte: Adicione o diâmetro do círculo ocidental ao do leste e subtraia o do sul, e você obterá o do norte. Fim. (Ahuja; Uegaki; Matsushita, 2006, p. 89)

Na inscrição acima, 1 sol era uma unidade de medida tradicional japonesa cujo valor é, aproximadamente, igual a 3 centímetros. As seis linhas descritas no teorema são os lados e diagonais do quadrilátero. A Figura 5 ilustra-nos tal inscrição. 


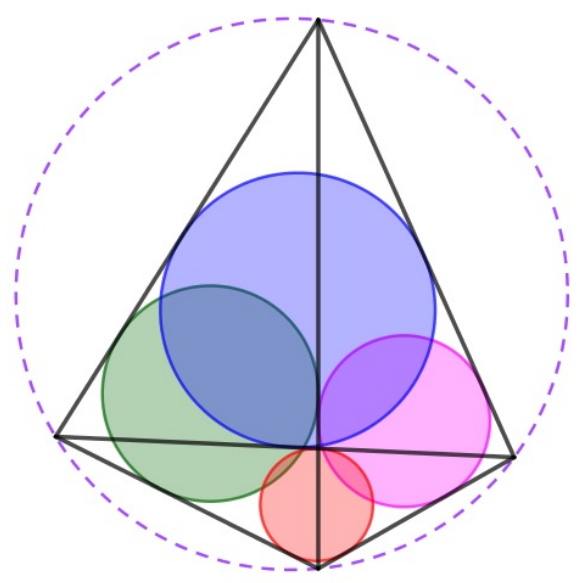

Figura 5: Uma possível ilustração para o Teorema Japonês.

É importante ressaltar que, durante quase toda Era Edo, o Japão foi uma sociedade completamente fechada para o mundo exterior. Contribuições importantes como as de Newton, Leibniz e Euler não chegaram ao conhecimento do povo japonês durante esse período. Somente no início do século 20 que documentos de matemáticos japoneses começaram a aparecer em periódicos ocidentais. Atualmente ainda existem aproximadamente 900 sangaku e acredita-se que milhares foram perdidos ao longo do tempo. Na Tabela 1 podemos observar a quantidade de sangaku existentes por prefeitura.

\begin{tabular}{|c|c|}
\hline Prefeitura & Quantidade de sangaku \\
\hline \hline Hokkaido & 0 \\
\hline Aomori & 3 \\
\hline Iwate & 102 \\
\hline Miyagi & 49 \\
\hline Akita & 8 \\
\hline Yamagata & 37 \\
\hline Fukushima & 111 \\
\hline Ibaraki & 21 \\
\hline Tochigi & 21 \\
\hline Gunma & 78 \\
\hline Saitama & 88 \\
\hline Chiba & 33 \\
\hline
\end{tabular}




\begin{tabular}{|c|c|}
\hline Prefeitura & Quantidade de sangaku \\
\hline Tōkyō & 16 \\
\hline Kanagawa & 6 \\
\hline Yamanashi & 5 \\
\hline Niigata & 27 \\
\hline Toyama & 17 \\
\hline Ishikawa & 16 \\
\hline Fukui & 23 \\
\hline Nagano & 54 \\
\hline Shizuoka & 7 \\
\hline Aichi & 16 \\
\hline Gifu & 8 \\
\hline Mie & 14 \\
\hline Shiga & 11 \\
\hline Kyōto & 17 \\
\hline Osaka & 13 \\
\hline Hyōgo & 27 \\
\hline Nara & 5 \\
\hline Wakayama & 1 \\
\hline Tottori & 0 \\
\hline Shimane & 0 \\
\hline Okayama & 25 \\
\hline Hiroshima & 4 \\
\hline Yamaguchi & 0 \\
\hline Tokushima & 0 \\
\hline Kagawa & 7 \\
\hline Ehime & 31 \\
\hline Kōchi & 0 \\
\hline Fukuoka & 8 \\
\hline Saga & 1 \\
\hline Nagasaki & 3 \\
\hline Kumamoto & 0 \\
\hline Oita & 1 \\
\hline Miyazaki & 0 \\
\hline Kagoshima & 0 \\
\hline Okinawa & 0 \\
\hline
\end{tabular}

Tabela 1: Quantidade de sangaku existentes por prefeitura.

Fonte: [11], p. 25.

O Teorema Japonês foi parte da minha pesquisa de mestrado Profmat [20], em parceria com meu orientador e professor Fábio Silva de Souza, na Faculdade de Formação de Professores - Uerj, São Gonçalo. Trata-se de um resultado muito interessante, como boa parte dos problemas contidos em sangaku japoneses, e cuja prova contém elementos de geometria bastante comuns na educação básica. 


\section{O Teorema Japonês para quadriláteros cíclicos convexos}

Nesta seção, mostraremos a prova registrada mais antiga do Teorema Japonês para quadriláteros que, segundo Ahuja [1], é atribuída a Tameyuki Yoshida [21]. Para os nossos propósitos, as seguintes definições serão importantes.

Definição 1. Um polígono é dito cíclico quando todos os seus vértices pertencem ao mesmo círculo. Nesse caso, o raio de tal círculo é chamado circunraio do polígono.

Definição 2. O inraio de um polígono é o raio de seu círculo inscrito.

Definição 3. Triangularizar um polígono a partir de um determinado vértice é traçar todas as diagonais possíveis do polígono cujo vértice em questão é extremidade.

Teorema 1 (Teorema Japonês para quadriláteros). Seja ABCD um quadrilátero cíclico convexo. Então a soma dos inraios dos triângulos resultantes de uma triangularização do quadrilátero $\mathrm{ABCD}$ independe da diagonal escolhida para formar essa triangularização.

Demonstração. Seja ABCD um quadrilátero cíclico convexo tal que $\mathrm{r}_{\mathrm{a}}, \mathrm{r}_{\mathrm{b}}, \mathrm{r}_{\mathrm{c}}$ e $\mathrm{r}_{\mathrm{d}}$ denotam, respectivamente, as medidas dos inraios dos triângulos ABD, BCA, CDB e DAC. Chamando tais triângulos, respectivamente, de $\mathrm{T}_{1}, \mathrm{~T}_{2}, \mathrm{~T}_{3}$ e $\mathrm{T}_{4}$, consideremos que $\mathrm{P}_{\mathrm{i}}, \mathrm{Q}_{\mathrm{i}}$ e $\mathrm{S}_{\mathrm{i}}$ são os pontos de tangência do círculo $\mathrm{C}_{\mathrm{i}}$ com $\mathrm{T}_{\mathrm{i}}$, onde $\mathrm{i}=1,2,3,4$.

Considerando uma primeira triangularização de ABCD, dada pela Figura 6, e também a expressão E, dada por

$$
\mathrm{E}=\overline{\mathrm{AB}}+\overline{\mathrm{CD}}-\overline{\mathrm{BC}}-\overline{\mathrm{AD}}
$$

podemos observar que

$$
\mathrm{E}=\left(\overline{\mathrm{AQ}_{1}}+\overline{\mathrm{BQ}_{1}}\right)+\left(\overline{\mathrm{CQ}_{3}}+\overline{\mathrm{DQ}_{3}}\right)-\left(\overline{\mathrm{BP}_{3}}+\overline{\mathrm{CP}_{3}}\right)-\left(\overline{\mathrm{AP}_{1}}+\overline{\mathrm{DP}_{1}}\right) .
$$

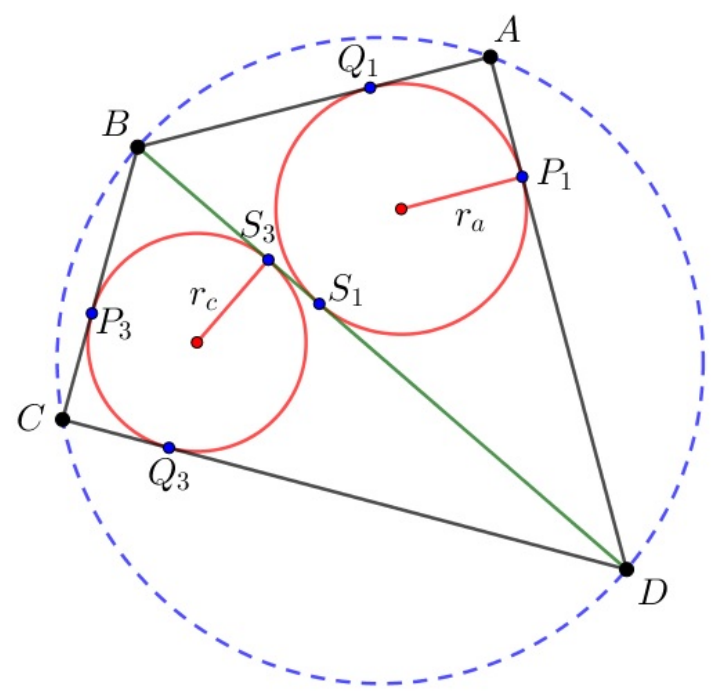

Figura 6: Primeira triangularização do quadrilátero ABCD. 
Utilizando a propriedade de congruência entre segmentos tangentes a um círculo, obtemos

$$
\overline{\mathrm{AQ}_{1}}=\overline{\mathrm{AP}_{1}}, \quad \overline{\mathrm{BQ}_{1}}=\overline{\mathrm{BS}_{1}}, \quad \overline{\mathrm{BP}_{3}}=\overline{\mathrm{BS}_{3}}, \quad \overline{\mathrm{CP}_{3}}=\overline{\mathrm{CQ}_{3}}, \overline{\mathrm{DQ}_{3}}=\overline{\mathrm{DS}_{3}}, \quad \text { e } \overline{\mathrm{DP}_{1}}=\overline{\mathrm{DS}_{1}},
$$

de forma que a expressão E, após algumas simplificações, pode ser reescrita como

$$
\mathrm{E}=\left(\overline{\mathrm{BS}_{1}}-\overline{\mathrm{BS}_{3}}\right)+\left(\overline{\mathrm{DS}_{3}}-\overline{\mathrm{DS}_{1}}\right)=\overline{\mathrm{S}_{1} \mathrm{~S}_{3}}+\overline{\mathrm{S}_{1} \mathrm{~S}_{3}},
$$

ou seja,

$$
\mathrm{E}=2 \cdot \overline{\mathrm{S}_{1} \mathrm{~S}_{3}}
$$

Por outro lado, considerando uma segunda triangularização para ABCD, dada pela Figura 7, obtemos

$$
\mathrm{E}=\left(\overline{\mathrm{AP}_{2}}+\overline{\mathrm{BP}_{2}}\right)+\left(\overline{\mathrm{CP}_{4}}+\overline{\mathrm{DP}_{4}}\right)-\left(\overline{\mathrm{BQ}_{2}}+\overline{\mathrm{CQ}_{2}}\right)-\left(\overline{\mathrm{AQ}_{4}}+\overline{\mathrm{DQ}_{4}}\right) .
$$

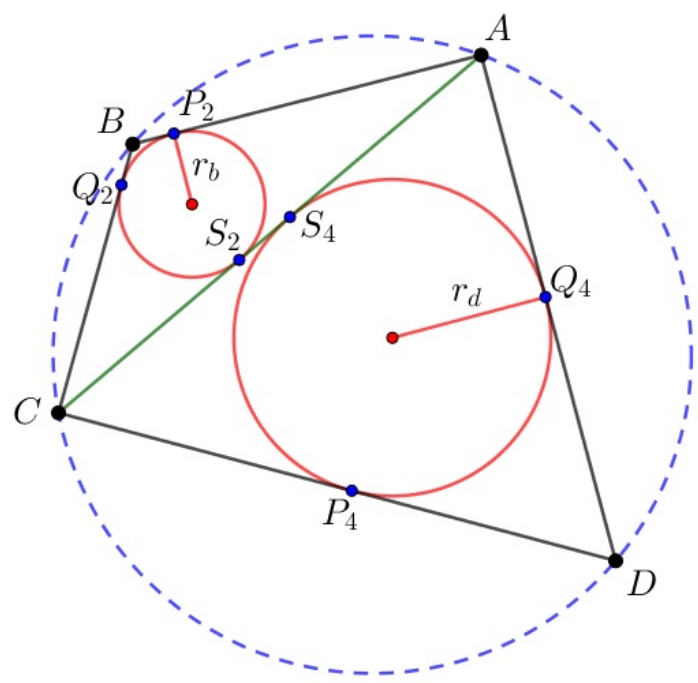

Figura 7: Segunda triangularização do quadrilátero ABCD.

Novamente, pela propriedade de congruência entre segmentos tangentes a um círculo, podemos escrever

$$
\overline{\mathrm{AP}_{2}}=\overline{\mathrm{AS}_{2}}, \quad \overline{\mathrm{BP}_{2}}=\overline{\mathrm{BQ}_{2}}, \quad \overline{\mathrm{CQ}_{2}}=\overline{\mathrm{CS}_{2}}, \quad \overline{\mathrm{CP}_{4}}=\overline{\mathrm{CS}_{4}}, \overline{\mathrm{DP}_{4}}=\overline{\mathrm{DQ}_{4}} \quad \text { e } \overline{\mathrm{AQ}_{4}}=\overline{\mathrm{AS}_{4}} \text {, }
$$

de maneira que E, após algumas simplificações, também pode ser reescrita como

$$
\mathrm{E}=\left(\overline{\mathrm{AS}_{2}}-\overline{\mathrm{AS}_{4}}\right)+\left(\overline{\mathrm{CS}_{4}}-\overline{\mathrm{CS}_{2}}\right)=\overline{\mathrm{S}_{2} \mathrm{~S}_{4}}+\overline{\mathrm{S}_{2} \mathrm{~S}_{4}},
$$

ou seja,

$$
\mathrm{E}=2 \cdot \overline{\mathrm{S}_{2} \mathrm{~S}_{4}}
$$


Comparando as Equações (1) e (2), obtemos

$$
2 \cdot \overline{\mathrm{S}_{1} \mathrm{~S}_{3}}=2 \cdot \overline{\mathrm{S}_{2} \mathrm{~S}_{4}},
$$

de onde segue que

$$
\overline{\mathrm{S}_{1} \mathrm{~S}_{3}}=\overline{\mathrm{S}_{2} \mathrm{~S}_{4}} .
$$

Os ângulos $\angle \mathrm{ACB}$ e $\angle \mathrm{ADB}$ são ambos inscritos no mesmo $\widehat{\mathrm{AB}}$ e, portanto, podemos considerar $\mathrm{A} \widehat{\mathrm{CB}}=\mathrm{AD} \mathrm{B}=2 \alpha$, para algum ângulo $\alpha \in(0, \pi / 2)$. Utilizando argumento análogo, escrevemos

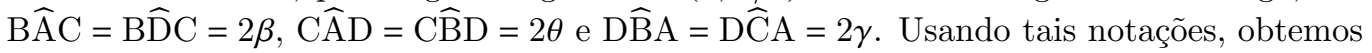

$$
\begin{aligned}
& \operatorname{tg} \alpha=\frac{\mathrm{r}_{\mathrm{b}}}{\overline{\mathrm{CS}_{2}}}=\frac{\mathrm{r}_{\mathrm{a}}}{\overline{\overline{\mathrm{DS}_{1}}}} \Longrightarrow \mathrm{r}_{\mathrm{b}} \cdot \overline{\mathrm{DS}_{1}}-\mathrm{r}_{\mathrm{a}} \cdot \overline{\mathrm{CS}_{2}}=0 \\
& \operatorname{tg} \beta=\frac{\mathrm{r}_{\mathrm{b}}}{\overline{\mathrm{AS}_{2}}}=\frac{\mathrm{r}_{\mathrm{c}}}{\overline{\overline{\mathrm{DS}_{3}}}} \Longrightarrow \mathrm{r}_{\mathrm{c}} \cdot \overline{\mathrm{AS}_{2}}-\mathrm{r}_{\mathrm{b}} \cdot \overline{\mathrm{DS}_{3}}=0, \\
& \operatorname{tg} \theta=\frac{\mathrm{r}_{\mathrm{d}}}{\overline{\mathrm{AS}_{4}}}=\frac{\mathrm{r}_{\mathrm{c}}}{\overline{\mathrm{BS}_{3}}} \Longrightarrow \mathrm{r}_{\mathrm{d}} \cdot \overline{\mathrm{BS}_{3}}-\mathrm{r}_{\mathrm{c}} \cdot \overline{\mathrm{AS}_{4}}=0 \\
& \text { e } \\
& \operatorname{tg} \gamma=\frac{\mathrm{r}_{\mathrm{d}}}{\overline{\mathrm{CS}_{4}}}=\frac{\mathrm{r}_{\mathrm{a}}}{\overline{\mathrm{BS}_{1}}} \Longrightarrow \mathrm{r}_{\mathrm{a}} \cdot \overline{\mathrm{CS}_{4}}-\mathrm{r}_{\mathrm{d}} \cdot \overline{\mathrm{BS}_{1}}=0 .
\end{aligned}
$$

Somando as equações acima, membro a membro, obtemos

$$
\mathrm{r}_{\mathrm{a}}\left(\overline{\mathrm{CS}_{4}}-\overline{\mathrm{CS}_{2}}\right)+\mathrm{r}_{\mathrm{c}}\left(\overline{\mathrm{AS}_{2}}-\overline{\mathrm{AS}_{4}}\right)-\mathrm{r}_{\mathrm{b}}\left(\overline{\mathrm{DS}_{3}}-\overline{\mathrm{DS}_{1}}\right)-\mathrm{r}_{\mathrm{d}}\left(\overline{\mathrm{BS}_{1}}-\overline{\mathrm{BS}_{3}}\right)=0 .
$$

Portanto,

$$
\mathrm{r}_{\mathrm{a}} \cdot \overline{\mathrm{S}_{2} \mathrm{~S}_{4}}+\mathrm{r}_{\mathrm{c}} \cdot \overline{\mathrm{S}_{2} \mathrm{~S}_{4}}-\mathrm{r}_{\mathrm{b}} \cdot \overline{\mathrm{S}_{1} \mathrm{~S}_{3}}-\mathrm{r}_{\mathrm{d}} \cdot \overline{\mathrm{S}_{1} \mathrm{~S}_{3}}=0,
$$

de onde segue

$$
\left(r_{a}+r_{c}\right) \cdot \overline{S_{2} S_{4}}=\left(r_{b}+r_{d}\right) \cdot \overline{S_{1} S_{3}} .
$$

Finalmente, utilizando (3), concluímos que

$$
r_{a}+r_{c}=r_{b}+r_{d} .
$$

Vale lembrar que existe uma caracterização bem conhecida dos quadriláteros cíclicos convexos quando olhamos para seus ângulos internos. Recorde que um quadrilátero convexo ABCD é cíclico se, e somente se, $\mathrm{DA} B+\mathrm{BCD}=\pi$ ou $\mathrm{B} \widehat{\mathrm{A}} \mathrm{C}=\mathrm{B} \widehat{\mathrm{DC}}$. Por outro lado, se duas triangularizações distintas de um quadrilátero convexo gerarem soma de inraios distintas, o Teorema 1 garante-nos que esse quadrilátero não é cíclico.

Na segunda parte do artigo [1] se encontram diversas outras provas interessantes para o Teorema 1. Um fato surpreendente é que o resultado obtido também é válido para um polígono cíclico convexo qualquer. Veremos isso na próxima seção. 


\section{O Teorema Japonês para polígonos cíclicos convexos}

Nesta seção iremos apresentar duas provas do Teorema Japonês para polígonos cíclicos convexos. A primeira delas foi dada por Gusman [8] e o autor utiliza basicamente o Teorema de Carnot para triângulos, que pode ser encontrado no artigo indicado ou, se o leitor preferir, nas páginas 193 e 194 de [16]. A segunda prova foi dada por Ahuja na segunda parte de seu artigo [1], e utiliza o Método da Indução Matemática, que pode ser encontrado na página 24 de [4]. Para enunciarmos o Teorema de Carnot de uma maneira mais resumida, adotaremos a seguinte convenção.

Uma determinada distância será negativa, quando nenhuma parte de seu segmento correspondente estiver contida no interior do respectivo polígono. Caso contrário, tal distância será positiva. As Figuras 8 e 9 possibilitam-nos visualizar tal convenção para triângulos.

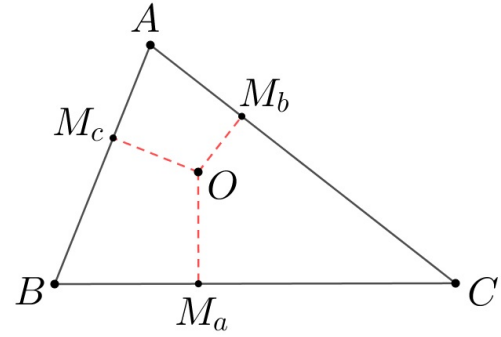

Figura 8: Distâncias $\overline{\mathrm{OM}_{\mathrm{a}}}, \overline{\mathrm{OM}_{\mathrm{b}}}$ e $\overline{\mathrm{OM}_{\mathrm{c}}}$ positivas.

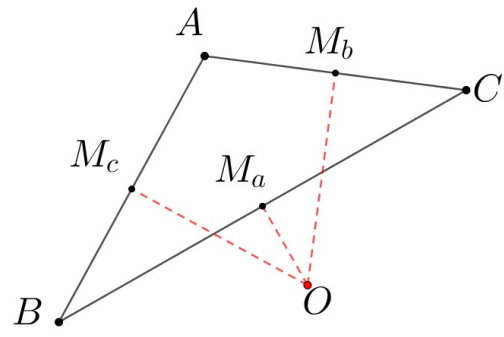

Figura 9: Distância $\overline{\mathrm{OM}_{\mathrm{a}}}$ negativa.

Lema 1 (Teorema de Carnot). Se ABC é um triângulo qualquer de inraio $\mathrm{r}$ e circunraio $\mathrm{R}$, então a soma das distâncias do circuncentro de $\mathrm{ABC}$ aos seus respectivos lados é igual a $\mathrm{r}+\mathrm{R}$.

Lema 2 (Método da Indução Matemática). Seja $\mathcal{P}(\mathrm{n})$ uma propriedade relativa ao número natural $\mathrm{n}$ e seja $\mathrm{n}_{0}$ um número natural. Suponhamos que

- $\mathcal{P}\left(\mathrm{n}_{0}\right)$ seja válida;

- Para todo $\mathrm{n} \geq \mathrm{n}_{0}$, a validez de $\mathcal{P}(\mathrm{n})$ implica a validez de $\mathcal{P}(\mathrm{n}+1)$.

Então $\mathcal{P}(\mathrm{n})$ é válida para todo $\mathrm{n} \geq \mathrm{n}_{0}$.

Teorema 2 (Teorema Japonês generalizado). Se triangularizarmos um polígono cíclico convexo a partir de um vértice qualquer, então a soma dos inraios dos triângulos resultantes dessa triangularização não dependerá do vértice escolhido.

Primeira demonstração. Seja $\mathrm{P}$ um polígono cíclico convexo com n lados e cujos vértices são $\mathrm{A}_{1}$, $\mathrm{A}_{2}, \cdots, \mathrm{A}_{\mathrm{n}}$, com $\mathrm{n} \geq 4$. Escolhendo um vértice qualquer para triangularizarmos $\mathrm{P}$, tal triangularização irá gerar um total de $(n-2)$ triângulos e isso se deve ao fato de que temos um total de $(\mathrm{n}-3)$ diagonais partindo do vértice escolhido e que tais diagonais determinam $(\mathrm{n}-3)+1$ triângulos interiores a P. A Figura 10 ilustra-nos um exemplo de triangularização para o referido polígono. 


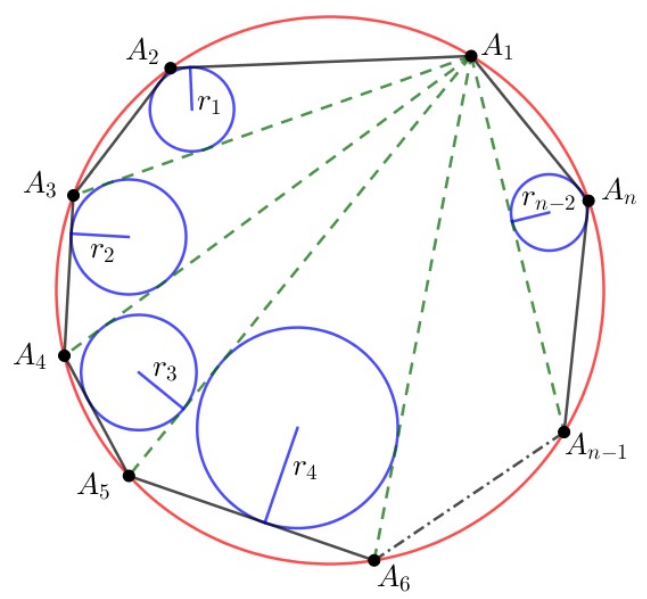

Figura 10: Triangularização de $\mathrm{P}$ a partir do vértice $\mathrm{A}_{1}$.

Sendo $\mathrm{O}$ o circuncentro do polígono, vamos definir a medida $\overline{\mathrm{OX}_{\mathrm{i}}}$ dada por

$$
\overline{\mathrm{OX}_{\mathrm{i}}}=\overline{\mathrm{OM}_{\mathrm{ai}}}+\overline{\mathrm{OM}_{\mathrm{bi}}}+\overline{\mathrm{OM}_{\mathrm{ci}}}
$$

onde $\overline{\mathrm{OM}_{\mathrm{ai}}}, \overline{\mathrm{OM}_{\mathrm{bi}}}$ e $\overline{\mathrm{OM}_{\mathrm{ci}}}$ são as distâncias de $\mathrm{O}$ até cada um dos lados do triângulo $\mathrm{T}_{\mathrm{i}}$ gerado pela triangularização de $\mathrm{P}$, com $\mathrm{i}=1,2, \cdots, \mathrm{n}-2$. Dessa forma obtemos

$$
\begin{aligned}
& \overline{\mathrm{OX}_{1}}=\overline{\mathrm{OM}_{\mathrm{a} 1}}+\overline{\mathrm{OM}_{\mathrm{b} 1}}+\overline{\mathrm{OM}_{\mathrm{c} 1}}, \\
& \overline{\mathrm{OX}_{2}}=\overline{\mathrm{OM}_{\mathrm{a} 2}}+\overline{\mathrm{OM}_{\mathrm{b} 2}}+\overline{\mathrm{OM}_{\mathrm{c} 2}}, \\
& \overline{\mathrm{OX}_{3}}=\overline{\mathrm{OM}_{\mathrm{a} 3}}+\overline{\mathrm{OM}_{\mathrm{b} 3}}+\overline{\mathrm{OM}_{\mathrm{c} 3}},
\end{aligned}
$$

$$
\overline{\mathrm{OX}_{\mathrm{n}-2}}=\overline{\mathrm{OM}_{\mathrm{a}(\mathrm{n}-2)}}+\overline{\mathrm{OM}_{\mathrm{b}(\mathrm{n}-2)}}+\overline{\mathrm{OM}_{\mathrm{c}(\mathrm{n}-2)}}
$$

Somando, membro a membro, as equações acima e utilizando o Lema 1 em cada triângulo $\mathrm{T}_{\mathrm{i}}$, de inraio $r_{i}$ e circunraio $R$, obtemos

$$
\sum_{i=1}^{n-2} \overline{\mathrm{OX}_{i}}=\sum_{i=1}^{\mathrm{n}-2}\left(\mathrm{r}_{\mathrm{i}}+\mathrm{R}\right)=\sum_{\mathrm{i}=1}^{\mathrm{n}-2} \mathrm{r}_{\mathrm{i}}+(\mathrm{n}-2) \cdot \mathrm{R}
$$

de modo que 


$$
\sum_{i=1}^{n-2} r_{i}=(2-n) \cdot R+\sum_{i=1}^{n-2} \overline{O_{i}}
$$

Observe que o termo $(2-n) \cdot R$ que aparece na Equação (4) é constante, pois tanto a quantidade de lados do polígono como a medida do circunraio $\mathrm{R}$ são bem determinadas. Mais ainda, pela definição de $\overline{\mathrm{OX}_{\mathrm{i}}}$, no somatório $\sum_{\mathrm{i}=1}^{\mathrm{n}-2} \overline{\mathrm{OX}_{\mathrm{i}}}$ cada perpendicular aos lados do polígono é contada uma única vez, enquanto que cada perpendicular às diagonais geradas pela triangularização de $\mathrm{P}$ é contada duas vezes, sendo uma delas positiva e outra negativa, conforme a convenção adotada para distâncias no Lema 1.

Por exemplo, na Figura 11, as distâncias $\overline{\mathrm{OM}_{\mathrm{a} 1}}$ e $\overline{\mathrm{OM}_{\mathrm{a} 2}}$ anulam-se. Já na Figura 12, os pares que se anulam são $\overline{\mathrm{OM}_{\mathrm{a} 1}} \operatorname{com} \overline{\mathrm{OM}_{\mathrm{a} 2}}$ e $\overline{\mathrm{OM}_{\mathrm{c} 2}} \operatorname{com} \overline{\mathrm{OM}_{\mathrm{c} 3}}$.

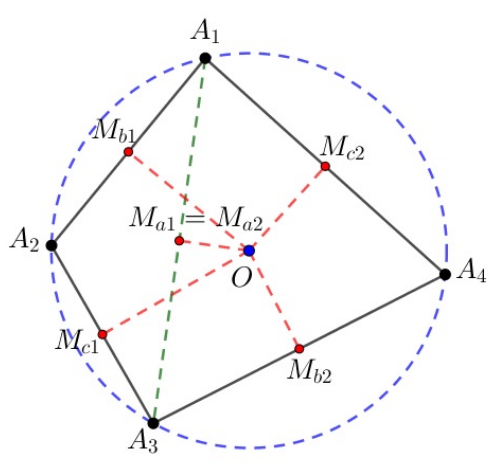

Figura 11: Teorema Japonês - caso n=4.

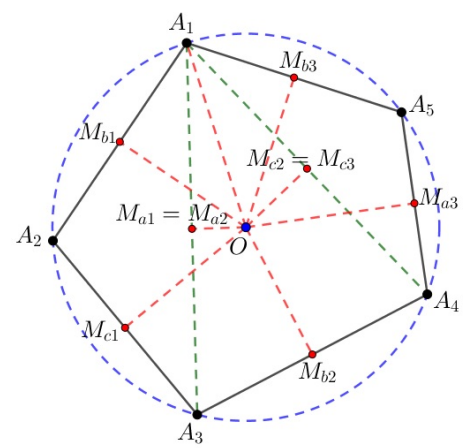

Figura 12: Teorema Japonês - caso $n=5$.

Dessa forma, todas as perpendiculares às diagonais irão se anular no somatório $\sum_{\mathrm{i}=1}^{\mathrm{n}-2} \overline{\mathrm{OX}_{\mathrm{i}}}$ sobrando apenas as perpendiculares aos lados, ou seja, as distâncias de $\mathrm{O}$ aos lados do polígono. Porém, tal soma também é uma constante, pois não depende do tipo de triangularização escolhida. Consequentemente,

$$
\sum_{i=1}^{n-2} r_{i}=\text { constante. }
$$

Segunda demonstração. Usaremos o Método da Indução no número de lados n do polígono $\mathrm{P}$. Vamos considerar que os vértices $\mathrm{A}_{1}, \mathrm{~A}_{2}, \cdots, \mathrm{A}_{\mathrm{n}}$ do polígono estejam dispostos ordenadamente no sentido anti-horário.

- $\mathcal{P}(4)$ é válida conforme Teorema 1 da Seção 2;

- Suponhamos que $\mathcal{P}(\mathrm{n})$ seja válida para todo $\mathrm{n} \geq 4$. Queremos mostrar a validade de $\mathcal{P}(\mathrm{n}+1)$. Para isso acrescentamos, sem perda de generalidade, o vértice $A_{n+1}$ entre os vértices $A_{n}$ e $A_{1}$ do polígono $\mathrm{P}$, de forma que $\mathrm{A}_{\mathrm{n}+1}$ esteja contido no mesmo círculo que os demais vértices. Com isso iremos gerar um novo polígono cíclico Q. A Figura 13 exemplifica tal argumento. 


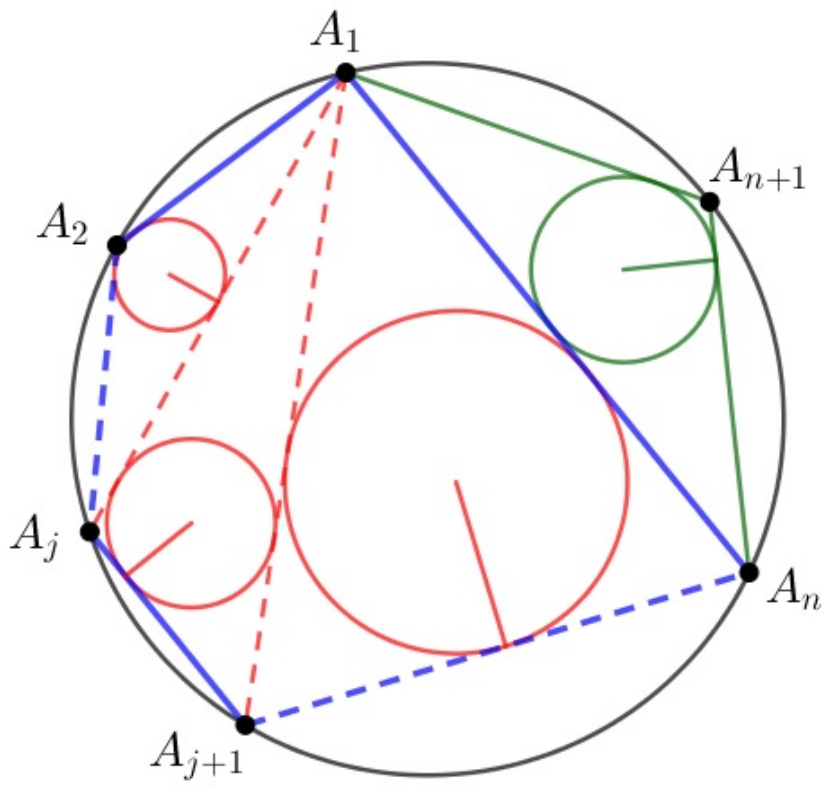

Figura 13: Triangularização dos polígonos $\mathrm{P}$ e $\mathrm{Q}$ a partir do vértice $\mathrm{A}_{1}$.

Sendo $S_{j}(P)$ e $S_{j}(Q)$, respectivamente, a soma dos inraios de todos os triângulos obtidos na triangularização dos polígonos $\mathrm{P}$ e $\mathrm{Q}$ a partir do vértice $\mathrm{A}_{\mathrm{j}}$, e considerando $\mathrm{r}$ [ABC] a medida do inraio de um determinado triângulo $\mathrm{ABC}$, se triangularizarmos $\mathrm{P}$ e $\mathrm{Q}$ a partir do vértice $\mathrm{A}_{1}$, então obtemos claramente que

$$
\mathrm{S}_{1}(\mathrm{Q})=\mathrm{S}_{1}(\mathrm{P})+\mathrm{r}\left[\mathrm{A}_{\mathrm{n}} \mathrm{A}_{\mathrm{n}+1} \mathrm{~A}_{1}\right]
$$

Agora, vamos triangularizar $\mathrm{P}$ e $\mathrm{Q}$ a partir de um vértice $\mathrm{A}_{\mathrm{j}}$ qualquer. Nesse caso, a soma dos inraios na triangularização do polígono $\mathrm{Q}$ será igual à soma dos inraios na triangularização do polígono $\mathrm{P}$ menos o inraio do triângulo $\mathrm{A}_{\mathrm{j}} \mathrm{A}_{\mathrm{n}} \mathrm{A}_{1}$, pois esse não aparece na triangularização de $\mathrm{Q}$, mais os inraios dos triângulos $A_{j} A_{n} A_{n+1}$ e $A_{j} A_{n+1} A_{1}$, pois esses não aparecem na triangularização de P. As figuras 14 e 15 permitem-nos visualizar essa situação. Logo,

$$
S_{j}(Q)=S_{j}(P)-r\left[A_{j} A_{n} A_{1}\right]+r\left[A_{j} A_{n} A_{n+1}\right]+r\left[A_{j} A_{n+1} A_{1}\right] .
$$




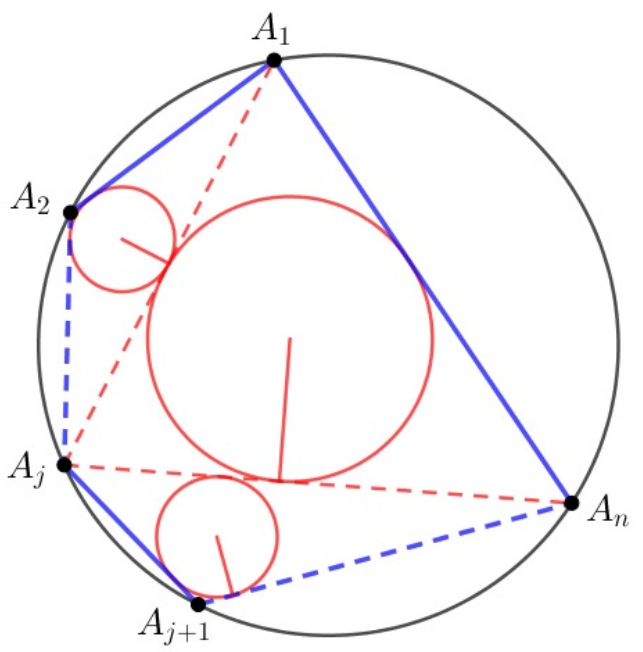

Figura 14: Triangularização de $\mathrm{P}$ por $\mathrm{A}_{\mathrm{j}}$.

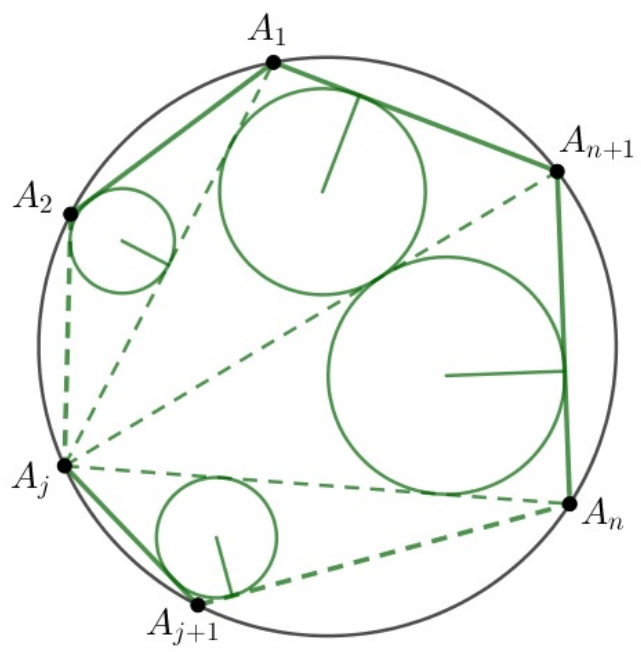

Figura 15: Triangularização de $\mathrm{Q}$ por $\mathrm{A}_{\mathrm{j}}$.

Considerando o quadrilátero $\mathrm{A}_{\mathrm{j}} \mathrm{A}_{\mathrm{n}} \mathrm{A}_{\mathrm{n}+1} \mathrm{~A}_{1}$, pelo Teorema 1 , temos que

$$
r\left[A_{j} A_{n} A_{n+1}\right]+r\left[A_{j} A_{n+1} A_{1}\right]=r\left[A_{j} A_{n} A_{1}\right]+r\left[A_{n} A_{n+1} A_{1}\right] .
$$

Substituindo (6) em (5), podemos escrever

$$
S_{j}(Q)=S_{j}(P)-r\left[A_{j} A_{n} A_{1}\right]+r\left[A_{j} A_{n} A_{1}\right]+r\left[A_{n} A_{n+1} A_{1}\right]
$$

de forma que

$$
S_{j}(Q)=S_{j}(P)+r\left[A_{n} A_{n+1} A_{1}\right]
$$

Pela hipótese de indução, a soma dos inraios do polígono $\mathrm{P}$ não depende do vértice escolhido, ou seja, $S_{j}(P)=S_{k}(P)$ sendo $A_{j}$ e $A_{k}$ vértices distintos. Somando $r\left[A_{n} A_{n+1} A_{1}\right]$ a ambos os membros, obtemos

$$
\mathrm{S}_{\mathrm{j}}(\mathrm{P})+\mathrm{r}\left[\mathrm{A}_{\mathrm{n}} \mathrm{A}_{\mathrm{n}+1} \mathrm{~A}_{1}\right]=\mathrm{S}_{\mathrm{k}}(\mathrm{P})+\mathrm{r}\left[\mathrm{A}_{\mathrm{n}} \mathrm{A}_{\mathrm{n}+1} \mathrm{~A}_{1}\right]
$$

e, utilizando a Equação (7), concluímos que

$$
\mathrm{S}_{\mathrm{j}}(\mathrm{Q})=\mathrm{S}_{\mathrm{k}}(\mathrm{Q})
$$

ou seja, tal propriedade também é válida para o polígono $\mathrm{Q}$, implicando a validez de $\mathcal{P}(\mathrm{n}+1)$. Pelo Lema $2, \mathcal{P}(\mathrm{n})$ é válida para todo n natural tal que $\mathrm{n} \geq 4$. 
Vale ressaltar que, utilizando os mesmos argumentos da primeira prova apresentada, verifica-se que o Teorema 2 também é válido quando triangularizamos o polígono de maneira arbitrária, ou seja, quando as diagonais não partem necessariamente de um mesmo vértice. A Figura 16 ilustra dois exemplos deste caso, quando $n=7$.
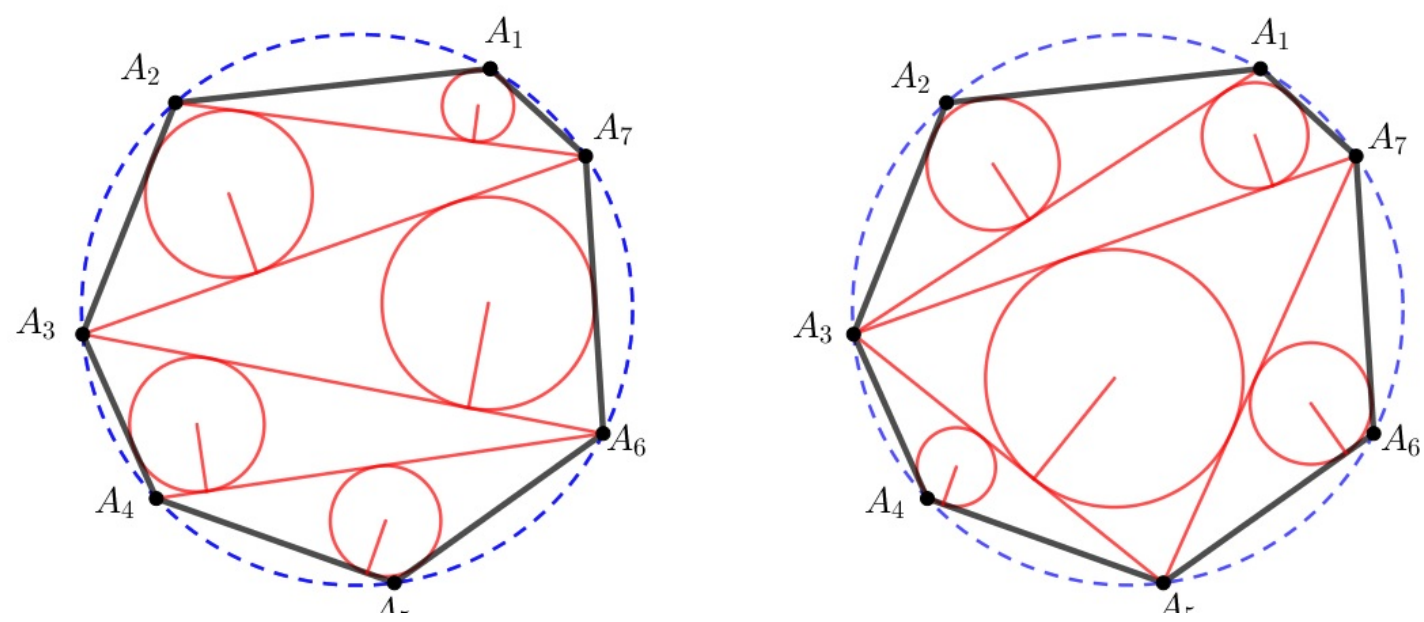

Figura 16: Duas possíveis triangularizações arbitrárias de P.

Podemos observar que há várias maneiras de triangularizar um polígono. E cada uma delas decompõe o polígono de formas distintas, e, portanto, os triângulos que aparecem em uma triangularização, em geral, são distintos dos de uma outra dada triangularização, e sendo assim, a priori, não é de se esperar que a soma dos inraios dos triângulos obtidos de uma triangularização qualquer seja sempre a mesma. Porém, surpreendentemente, tal soma independe da triangularização, e é isso que nos diz o Teorema Japonês.

\section{Duas propriedades para quadriláteros cíclicos convexos}

Uma primeira aplicação do Teorema Japonês pode ser encontrada em [15] e refere-se a uma propriedade para quadriláteros cíclicos convexos que envolve a diagonal de triangularização e os inraios dos triângulos obtidos. Em sua demonstração, iremos utilizar o seguinte lema cuja prova também se encontra na referência citada. As Figuras 6 e 7 podem ajudar na visualização de tal propriedade.

Lema 3. Seja ABCD um quadrilátero cíclico convexo de diagonais $\mathrm{AC}$ e BD. Se $\mathrm{r}_{\mathrm{a}}, \mathrm{r}_{\mathrm{b}}, \mathrm{r}_{\mathrm{c}}$ e $\mathrm{r}_{\mathrm{d}}$ denotam, respectivamente, os inraios dos triângulos $\mathrm{ABD}, \mathrm{BCA}, \mathrm{CDB}$ e $\mathrm{DAC}$ obtidos nas duas triangularizações do quadrilátero $\mathrm{ABCD}$, então

$$
\mathrm{r}_{\mathrm{a}} \cdot \mathrm{r}_{\mathrm{c}} \cdot \overline{\mathrm{AC}}=\mathrm{r}_{\mathrm{b}} \cdot \mathrm{r}_{\mathrm{d}} \cdot \overline{\mathrm{BD}}
$$

Teorema 3. Se ABCD é um quadrilátero cíclico convexo, então o produto entre a diagonal de triangularização e a soma dos inversos dos inraios dos triângulos obtidos não depende da diagonal escolhida. 
Demonstração. Consideremos inicialmente que $\overline{\mathrm{AC}}=\mathrm{x}$ e $\overline{\mathrm{BD}}=\mathrm{y}$. O produto da diagonal BD pela soma dos inversos dos inraios $\mathrm{r}_{\mathrm{a}}$ e $\mathrm{r}_{\mathrm{c}}$ dos triângulos obtidos pode ser escrito como

$$
\mathrm{y} \cdot\left(\frac{1}{\mathrm{r}_{\mathrm{a}}}+\frac{1}{\mathrm{r}_{\mathrm{c}}}\right)=\mathrm{y} \cdot\left(\frac{\mathrm{r}_{\mathrm{a}}+\mathrm{r}_{\mathrm{c}}}{\mathrm{r}_{\mathrm{a}} \cdot \mathrm{r}_{\mathrm{c}}}\right)
$$

Pelo Lema 3, podemos escrever

$$
\mathrm{r}_{\mathrm{a}} \cdot \mathrm{r}_{\mathrm{c}}=\frac{\mathrm{y}}{\mathrm{x}} \cdot \mathrm{r}_{\mathrm{b}} \cdot \mathrm{r}_{\mathrm{d}}
$$

de forma que

$$
y \cdot\left(\frac{r_{a}+r_{c}}{r_{a} \cdot r_{c}}\right)=y \cdot\left[\frac{x \cdot\left(r_{a}+r_{c}\right)}{y \cdot r_{b} \cdot r_{d}}\right]=x \cdot\left(\frac{r_{a}+r_{c}}{r_{b} \cdot r_{d}}\right) .
$$

Utilizando o Teorema 1, obtemos

$$
\mathrm{r}_{\mathrm{a}}+\mathrm{r}_{\mathrm{c}}=\mathrm{r}_{\mathrm{b}}+\mathrm{r}_{\mathrm{d}}
$$

de onde segue

$$
y \cdot\left(\frac{1}{r_{a}}+\frac{1}{r_{c}}\right)=y \cdot\left(\frac{r_{a}+r_{c}}{r_{a} \cdot r_{c}}\right)=x \cdot\left(\frac{r_{a}+r_{c}}{r_{b} \cdot r_{d}}\right)=x \cdot\left(\frac{r_{b}+r_{d}}{r_{b} \cdot r_{d}}\right)=x \cdot\left(\frac{1}{r_{b}}+\frac{1}{r_{d}}\right) .
$$

Para apresentarmos uma outra aplicação do Teorema Japonês, precisamos nos lembrar que todo triângulo ABC possui três círculos ex-inscritos, ou seja, círculos que são tangentes externamente a um de seus lados e às retas suportes dos outros dois.

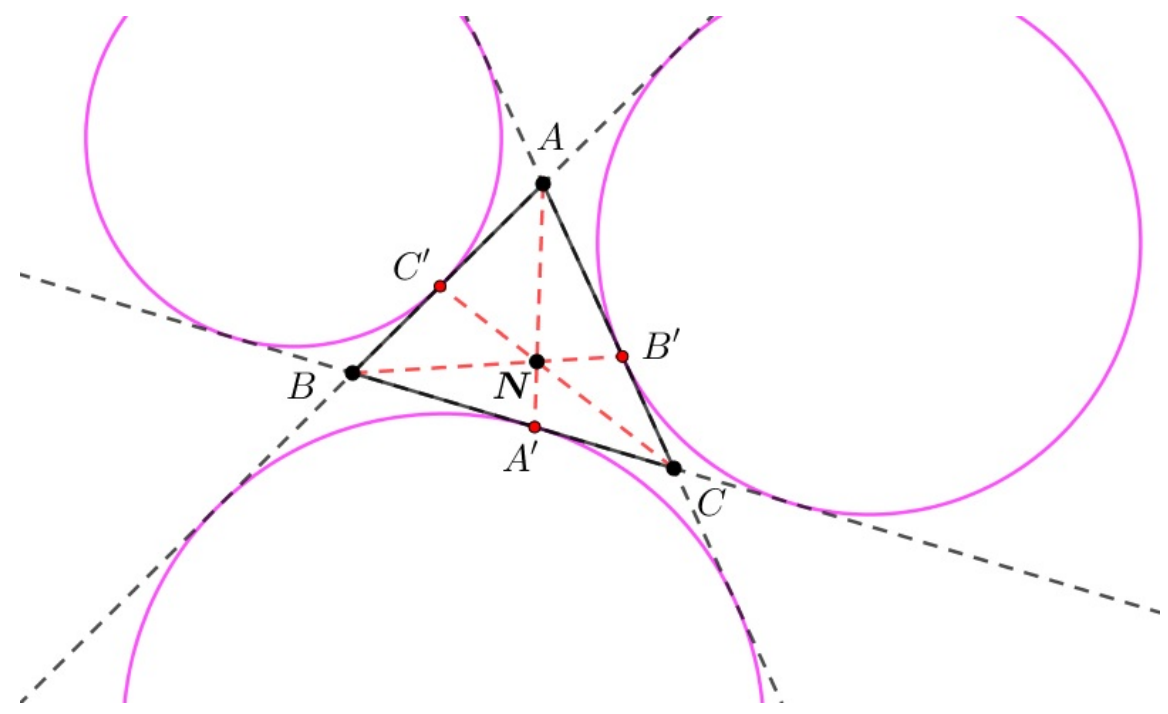

Figura 17: Ponto de Nagel de um triângulo ABC. 
Sendo $\mathrm{A}^{\prime}, \mathrm{B}^{\prime}$ e $\mathrm{C}^{\prime}$ os pontos de tangência dos referidos círculos, respectivamente, com os lados $\mathrm{BC}, \mathrm{AC}$ e $\mathrm{AB}$, pode-se mostrar, utilizando o Teorema 4 a seguir, que as cevianas $\mathrm{AA}^{\prime}, \mathrm{BB}^{\prime}$ e $\mathrm{CC}^{\prime}$ intersectam-se em um mesmo ponto, que denotaremos por N. Tal ponto é conhecido na literatura como o ponto de Nagel do triângulo ABC, devido ao matemático alemão do século XIX Christian Heinrich von Nagel (1803-1882). A prova do Teorema 4 pode ser encontrada nas páginas 20, 21 e 22 de [20]. Na Figura 17 podemos observar um exemplo de construção do referido ponto.

Teorema 4 (Teorema de Ceva). As três cevianas de um triângulo $\mathrm{ABC}$ são concorrentes se, e somente se,

$$
\frac{\overline{\mathrm{BP}}}{\overline{\mathrm{PC}}} \cdot \frac{\overline{\mathrm{CQ}}}{\overline{\mathrm{QA}}} \cdot \frac{\overline{\mathrm{AR}}}{\overline{\mathrm{RB}}}=1,
$$

onde $\mathrm{P}, \mathrm{Q}$ e $\mathrm{R}$ são, respectivamente, os pés de tais cevianas relativas aos lados $\mathrm{BC}, \mathrm{AC}$ e $\mathrm{AB}$.

Uma segunda aplicação do Teorema Japonês está associada a outra propriedade para quadriláteros cíclicos convexos que relaciona a soma das distâncias do circuncentro do quadrilátero aos pontos de Nagel dos triângulos obtidos em cada triangularização. Para os nossos propósitos o seguinte lema, que pode ser encontrado nas páginas 113 e 114 de [3] ou nas páginas 86 a 89 de [20], será importante.

Lema 4. Seja ABC um triângulo de circunraio $\mathrm{R}$ e inraio r. Se $\mathrm{O}$ e $\mathrm{N}$ denotam, respectivamente, o circuncentro e o ponto de Nagel do triângulo ABC, então

$$
\overline{\mathrm{ON}}=\mathrm{R}-2 \mathrm{r} .
$$

Teorema 5. Seja ABCD um quadrilátero cíclico convexo de circuncentro O. Se $\mathrm{N}_{\mathrm{a}}, \mathrm{N}_{\mathrm{b}}, \mathrm{N}_{\mathrm{c}}$ e $\mathrm{N}_{\mathrm{d}}$ denotam, respectivamente, os pontos de Nagel dos triângulos $\mathrm{ABD}, \mathrm{BCA}, \mathrm{CDB}$ e $\mathrm{DAC}$, então

$$
\overline{\mathrm{ON}_{\mathrm{a}}}+\overline{\mathrm{ON}_{\mathrm{c}}}=\overline{\mathrm{ON}_{\mathrm{b}}}+\overline{\mathrm{ON}_{\mathrm{d}}}
$$

Demonstração. Pelo Lema 4, podemos escrever

$$
\overline{\mathrm{ON}_{\mathrm{a}}}+\overline{\mathrm{ON}_{\mathrm{c}}}=\mathrm{R}-2 \mathrm{r}_{\mathrm{a}}+\mathrm{R}-2 \mathrm{r}_{\mathrm{c}}=2 \mathrm{R}-2 \cdot\left(\mathrm{r}_{\mathrm{a}}+\mathrm{r}_{\mathrm{c}}\right) \text {. }
$$

Por outro lado, usando o Teorema 1, obtemos

$$
\mathrm{r}_{\mathrm{a}}+\mathrm{r}_{\mathrm{c}}=\mathrm{r}_{\mathrm{b}}+\mathrm{r}_{\mathrm{d}}
$$

de onde segue

$$
\overline{\mathrm{ON}_{\mathrm{a}}}+\overline{\mathrm{ON}_{\mathrm{c}}}=2 \mathrm{R}-2\left(\mathrm{r}_{\mathrm{b}}+\mathrm{r}_{\mathrm{d}}\right)=\mathrm{R}-2 \mathrm{r}_{\mathrm{b}}+\mathrm{R}-2 \mathrm{r}_{\mathrm{d}}=\overline{\mathrm{ON}_{\mathrm{b}}}+\overline{\mathrm{ON}_{\mathrm{d}}}
$$

O Teorema 5 afirma que, para qualquer quadrilátero cíclico convexo, a soma das distâncias de seu circuncentro $\mathrm{O}$ até os pontos de Nagel dos triângulos obtidos em uma triangularização não depende da triangularização escolhida. As figuras 18 e 19 ilustram esse resultado onde a soma dos segmentos em vermelho é sempre igual à soma dos segmentos em azul. 


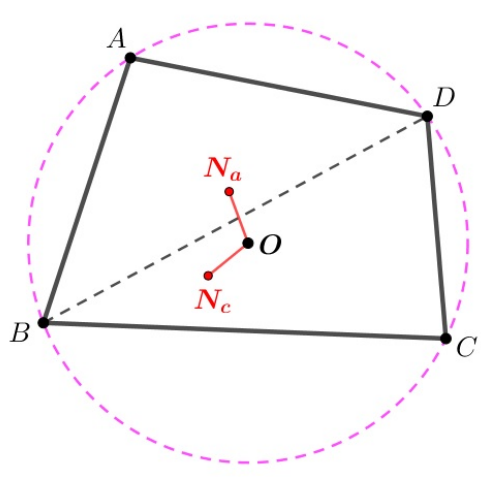

Figura 18: Distâncias $\overline{\mathrm{ON}_{\mathrm{a}}}$ e $\overline{\mathrm{ON}_{\mathrm{c}}}$.

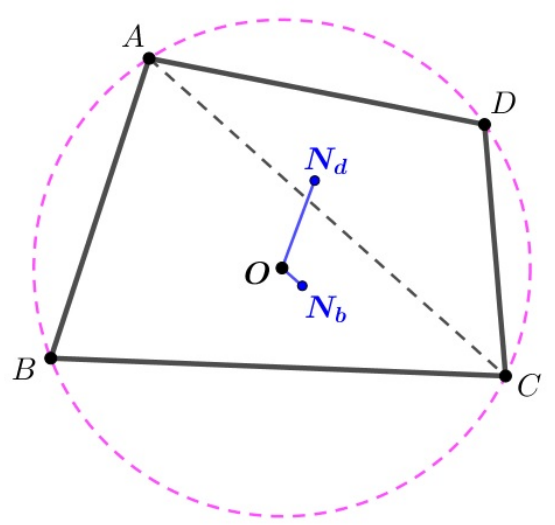

Figura 19: Distâncias $\overline{\mathrm{ON}_{\mathrm{b}}}$ e $\overline{\mathrm{ON}_{\mathrm{d}}}$.

\section{Um limite interessante}

No ano de 2013, Richeson [17] mostrou que o Teorema 2 também é válido para polígonos cíclicos não convexos. Nesse mesmo artigo, ele utilizou o referido teorema para visualizar de maneira simples a convergência da série

$$
\sum_{i=1}^{n-2} r_{i}
$$

que representa a soma de todos os inraios dos triângulos obtidos ao triangularizarmos um polígono cíclico convexo P. O Teorema 2 afirma que tal soma é sempre constante para P. Mas teríamos um limite para ela quando o número de lados do polígono for tão grande quanto desejarmos?

Para responder a essa pergunta, partimos da Equação (4). Dela obtemos que

$$
\sum_{i=1}^{n-2} r_{i}=(2-n) \cdot R+\sum_{i=1}^{n-2} \overline{\mathrm{OX}_{i}}
$$

onde $\overline{\mathrm{OX}_{\mathrm{i}}}$ é a soma das distâncias do circuncentro $\mathrm{O}$ do polígono $\mathrm{P}$ aos lados do triângulo $\mathrm{T}_{\mathrm{i}}$, $\mathrm{i}=1,2,3, \cdots, \mathrm{n}-2$, obtido na triangularização de $\mathrm{P}$. Nesse momento pode ser útil ao leitor observar as Figuras 11 e 12 que exemplificam, respectivamente, os casos em que $\mathrm{n}=4$ e $\mathrm{n}=5$.

Pelos argumentos apresentados na primeira demonstração do Teorema 2, as distâncias aos lados dos triângulos que são diagonais do polígono irão se cancelar conforme a convenção adotada para distâncias no Lema 1. As únicas contribuições para $\sum_{\mathrm{i}=1}^{\mathrm{n}-2} \overline{\mathrm{OX}_{\mathrm{i}}}$ são dadas pelas distâncias $\mathrm{d}_{\mathrm{i}}$, com $\mathrm{i}=1,2, \cdots, \mathrm{n}$, do circuncentro $\mathrm{O}$ a cada um dos lados $\mathrm{l}_{\mathrm{i}}$ do polígono. Nesse caso, podemos reescrever a Equação (4) como

$$
\sum_{i=1}^{n-2} r_{i}=(2-n) \cdot R+\sum_{i=1}^{n} d_{i}
$$

Cada lado $l_{\mathrm{i}}$ do polígono está associado a um ângulo central $\theta_{\mathrm{i}}$. Se $0 \leq \theta_{\mathrm{i}} \leq \pi$, então $\mathrm{d}_{\mathrm{i}}=\mathrm{R} \cdot \cos \left(\frac{\theta_{\mathrm{i}}}{2}\right)$. Por outro lado, se $\pi<\theta_{\mathrm{i}} \leq 2 \pi$, então $\mathrm{d}_{\mathrm{i}}$ será negativa e pode ser escrita $\operatorname{como} \mathrm{d}_{\mathrm{i}}=-\mathrm{R} \cdot \cos \phi$, para 
algum ângulo $\phi \in[0, \pi / 2)$, de forma que

$$
\phi+\frac{\theta_{\mathrm{i}}}{2}=\pi
$$

Logo,

$$
\mathrm{d}_{\mathrm{i}}=-\mathrm{R} \cdot \cos \left(\pi-\frac{\theta_{\mathrm{i}}}{2}\right)=\mathrm{R} \cdot \cos \left(\frac{\theta_{\mathrm{i}}}{2}\right)
$$

de onde segue que

$$
\mathrm{d}_{\mathrm{i}}=\mathrm{R} \cdot \cos \left(\frac{\theta_{\mathrm{i}}}{2}\right)
$$

para todo ângulo $\theta_{\mathrm{i}} \in[0,2 \pi]$. Podemos observar esses dois casos nas Figuras 20 e 21 .

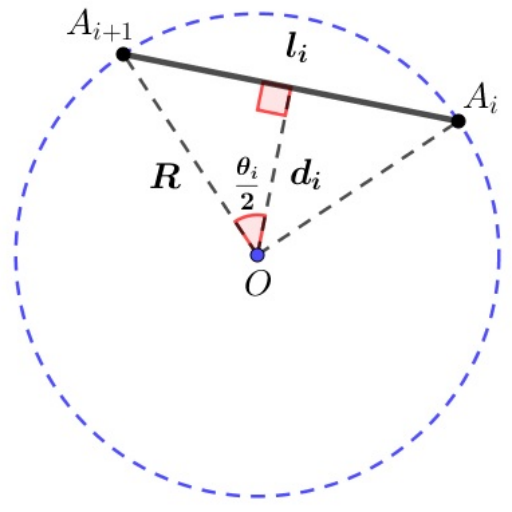

Figura 20: Caso $0 \leq \theta_{\mathrm{i}} \leq \pi$.

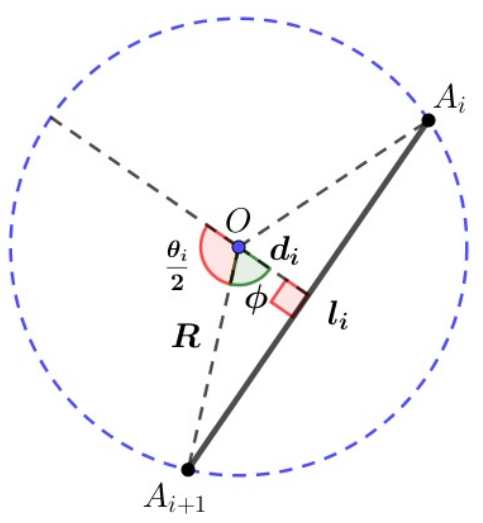

Figura 21: Caso $\pi<\theta_{\mathrm{i}} \leq 2 \pi$.

Substituindo (9) em (8) obtemos

$$
\sum_{i=1}^{n-2} r_{i}=(2-n) \cdot R+\sum_{i=1}^{n} R \cdot \cos \left(\frac{\theta_{i}}{2}\right)
$$

de forma que

$$
\sum_{i=1}^{n-2} r_{i}=R \cdot\left[2-n+\sum_{i=1}^{n} \cos \left(\frac{\theta_{i}}{2}\right)\right]
$$

Considerando $\mathcal{P}$ o espaço de todos os polígonos convexos com n lados, inscritos em um mesmo círculo de raio $\mathrm{R}$, e f : $\mathcal{P} \rightarrow \mathbb{R}$ uma função dada por

$$
f\left(P_{n}\right)=\sum_{i=1}^{n-2} r_{i}
$$

onde $\mathrm{P}_{\mathrm{n}}$ é um polígono desse espaço, temos que

$$
\mathrm{f}\left(\mathrm{P}_{\mathrm{n}}\right)=\mathrm{f}\left(\theta_{1}, \theta_{2}, \cdots, \theta_{\mathrm{n}}\right)=\mathrm{R} \cdot\left[2-\mathrm{n}+\sum_{\mathrm{i}=1}^{\mathrm{n}} \cos \left(\frac{\theta_{\mathrm{i}}}{2}\right)\right]
$$


é uma função nas variáveis $\theta_{1}, \theta_{2}, \cdots, \theta_{\mathrm{n}}$, se $\mathrm{n}$ for fixado. Repare que $\mathrm{f}$ é limitada por $2 \mathrm{R}$ que é justamente o diâmetro do círculo que circunscreve o polígono $\mathrm{P}_{\mathrm{n}}$. De fato, cada cosseno que aparece no somatório admite 1 como valor máximo, e, consequentemente,

$$
\mathrm{f}\left(\theta_{1}, \theta_{2}, \cdots, \theta_{\mathrm{n}}\right) \leq \mathrm{R} \cdot(2-\mathrm{n}+\mathrm{n} \cdot 1)=\mathrm{R} \cdot(2-\mathrm{n}+\mathrm{n})=2 \mathrm{R} .
$$

Para determinarmos o valor máximo da função f, utilizaremos o seguinte resultado, que pode ser encontrado na página 952 de [18] ou nas páginas 171 e 172 de [12], e é atribuído ao matemático franco-italiano Joseph-Louis Lagrange (1736-1813). Recordamos que $\nabla F$ denota o vetor gradiente de uma dada função F, ou seja,

$$
\nabla \mathrm{F}\left(\mathrm{x}_{1}, \mathrm{x}_{2}, \cdots, \mathrm{x}_{\mathrm{n}}\right)=\left(\frac{\partial \mathrm{F}}{\partial \mathrm{x}_{1}}, \frac{\partial \mathrm{F}}{\partial \mathrm{x}_{2}}, \cdots, \frac{\partial \mathrm{F}}{\partial \mathrm{x}_{\mathrm{n}}}\right) .
$$

Lema 5 (Método do Multiplicador de Lagrange). Seja f uma função diferenciável em um aberto U. Seja g uma função de classe $\mathrm{C}^{1}$ nesse mesmo aberto, tal que $\nabla \mathrm{g} \neq \overrightarrow{0}$ para todo $\left(\mathrm{x}_{1}, \mathrm{x}_{2}, \cdots, \mathrm{x}_{\mathrm{n}}\right) \in \mathrm{V}$, onde $\mathrm{V}=\left\{\left(\mathrm{x}_{1}, \mathrm{x}_{2}, \cdots, \mathrm{x}_{\mathrm{n}}\right) \in \mathrm{U} \mid \mathrm{g}\left(\mathrm{x}_{1}, \mathrm{x}_{2}, \cdots, \mathrm{x}_{\mathrm{n}}\right)=\mathrm{c}, \mathrm{c} \in \mathbb{R}\right\}$. Para determinar os valores máximo e mínimo de $\mathrm{f}\left(\mathrm{x}_{1}, \mathrm{x}_{2}, \cdots, \mathrm{x}_{\mathrm{n}}\right)$, sujeita à restrição $\mathrm{g}\left(\mathrm{x}_{1}, \mathrm{x}_{2}, \cdots, \mathrm{x}_{\mathrm{n}}\right)=\mathrm{c}$ (supondo que esses valores extremos existam):

- Determine todos os valores de $\mathrm{x}_{1}, \mathrm{x}_{2}, \cdots, \mathrm{x}_{\mathrm{n}}$ e $\lambda$ tais que

$$
\begin{gathered}
\nabla \mathrm{f}\left(\mathrm{x}_{1}, \mathrm{x}_{2}, \cdots, \mathrm{x}_{\mathrm{n}}\right)=\lambda \cdot \nabla \mathrm{g}\left(\mathrm{x}_{1}, \mathrm{x}_{2}, \cdots, \mathrm{x}_{\mathrm{n}}\right) \\
e \\
\mathrm{~g}\left(\mathrm{x}_{1}, \mathrm{x}_{2}, \cdots, \mathrm{x}_{\mathrm{n}}\right)=\mathrm{c} ;
\end{gathered}
$$

- Calcule $\mathrm{f}$ em todos os pontos $\left(\mathrm{x}_{1}, \mathrm{x}_{2}, \cdots, \mathrm{x}_{\mathrm{n}}\right)$ que resultam do passo anterior. O maior desses valores será o valor máximo de f, e o menor será o valor mínimo de f. O número real $\lambda$ é chamado multiplicador de Lagrange.

Consideremos as funções $\mathrm{f}$ e g, dadas por

$$
\mathrm{f}\left(\theta_{1}, \theta_{2}, \cdots, \theta_{\mathrm{n}}\right)=\mathrm{R} \cdot\left[2-\mathrm{n}+\sum_{\mathrm{i}=1}^{\mathrm{n}} \cos \left(\frac{\theta_{\mathrm{i}}}{2}\right)\right] \quad \text { e } \mathrm{g}\left(\theta_{1}, \theta_{2}, \cdots, \theta_{\mathrm{n}}\right)=\theta_{1}+\theta_{2}+\cdots+\theta_{\mathrm{n}} .
$$

Utilizando o Lema 5 para a função f na restrição

$$
\mathrm{g}\left(\theta_{1}, \theta_{2}, \cdots, \theta_{\mathrm{n}}\right)=2 \pi
$$

obtemos

$$
\nabla \mathrm{f}=\left(-\frac{\mathrm{R}}{2} \operatorname{sen}\left(\frac{\theta_{1}}{2}\right),-\frac{\mathrm{R}}{2} \operatorname{sen}\left(\frac{\theta_{2}}{2}\right), \cdots,-\frac{\mathrm{R}}{2} \operatorname{sen}\left(\frac{\theta_{\mathrm{n}}}{2}\right)\right) \quad \text { e } \quad \nabla \mathrm{g}=(1,1, \cdots, 1),
$$

de forma que $\nabla \mathrm{f}\left(\theta_{1}, \theta_{2}, \cdots, \theta_{\mathrm{n}}\right)=\lambda \cdot \nabla \mathrm{g}\left(\theta_{1}, \theta_{2}, \cdots, \theta_{\mathrm{n}}\right)$ leva-nos à seguinte sequência de igualdades

$$
-\frac{\mathrm{R}}{2} \operatorname{sen}\left(\frac{\theta_{1}}{2}\right)=-\frac{\mathrm{R}}{2} \operatorname{sen}\left(\frac{\theta_{2}}{2}\right)=\cdots=-\frac{\mathrm{R}}{2} \operatorname{sen}\left(\frac{\theta_{\mathrm{n}}}{2}\right)=\lambda,
$$


ou seja,

$$
\operatorname{sen}\left(\frac{\theta_{1}}{2}\right)=\operatorname{sen}\left(\frac{\theta_{2}}{2}\right)=\cdots=\operatorname{sen}\left(\frac{\theta_{\mathrm{n}}}{2}\right) .
$$

Afirmamos que o valor máximo $f_{M}$ da função $f$ ocorre para $\theta_{1}=\theta_{2}=\cdots=\theta_{\mathrm{n}}$. De fato, se $\theta_{\mathrm{i}}<\theta_{\mathrm{j}}$, para $\mathrm{i} \neq \mathrm{j}$, então

$$
\operatorname{sen}\left(\frac{\theta_{\mathrm{i}}}{2}\right)=\operatorname{sen}\left(\frac{\theta_{\mathrm{j}}}{2}\right)
$$

leva-nos a

$$
\frac{\theta_{\mathrm{j}}}{2}=\pi-\frac{\theta_{\mathrm{i}}}{2}
$$

de maneira que

$$
\theta_{\mathrm{i}}+\theta_{\mathrm{j}}=2 \pi
$$

Mas isso nos leva a um absurdo pois, o polígono $\mathrm{P}_{\mathrm{n}}$, nesse caso, seria representado por um ponto ou um segmento. Em ambos os casos temos $\mathrm{f}\left(\mathrm{P}_{\mathrm{n}}\right)=0$. Logo, pelo Lema 5, o valor máximo $\mathrm{f}_{\mathrm{M}}$ ocorre quando $\theta_{1}=\theta_{2}=\cdots=\theta_{\mathrm{n}}$, ou seja, $\mathrm{P}_{\mathrm{n}}$ é regular e cada ângulo central $\theta_{\mathrm{i}}$, com $\mathrm{i}=1,2, \cdots, \mathrm{n}$, pode ser escrito como

$$
\theta_{\mathrm{i}}=\frac{2 \pi}{\mathrm{n}}
$$

Substituindo (11) em (10), obtemos

$$
\mathrm{f}_{\mathrm{M}}\left(\mathrm{P}_{\mathrm{n}}\right)=\mathrm{R} \cdot\left[2-\mathrm{n}+\mathrm{n} \cdot \cos \left(\frac{2 \pi}{2 \mathrm{n}}\right)\right],
$$

de onde segue que $\mathrm{f}_{\mathrm{M}}$ depende somente do número de lados do polígono, ou seja,

$$
\mathrm{f}_{\mathrm{M}}(\mathrm{n})=\mathrm{R} \cdot\left\{2+\mathrm{n} \cdot\left[\cos \left(\frac{\pi}{\mathrm{n}}\right)-1\right]\right\}
$$

Podemos olhar $\mathrm{f}_{\mathrm{M}}$ como uma sequência de números reais onde o n-ésimo termo é dado pela igualdade acima. A sequência $f_{M}$ é crescente para $n>2$ pois $\cos (\pi / n)$ é crescente neste intervalo. Pelo fato de a função f ser limitada por $2 \mathrm{R}$, temos que $\mathrm{f}_{\mathrm{M}}$ é convergente. Vamos calcular o limite de $\mathrm{f}_{\mathrm{M}}$ quando $\mathrm{n}$ tende ao infinito.

Após algumas manipulações algébricas, podemos reescrever (12) como

$$
\mathrm{f}_{\mathrm{M}}(\mathrm{n})=\mathrm{R} \cdot\left[2-\pi \cdot \frac{\operatorname{sen}\left(\frac{\pi}{\mathrm{n}}\right)}{\left(\frac{\pi}{\mathrm{n}}\right)} \cdot \frac{\operatorname{sen}\left(\frac{\pi}{\mathrm{n}}\right)}{\cos \left(\frac{\pi}{\mathrm{n}}\right)+1}\right]
$$

Quando $n$ tende para o infinito, temos que $\frac{\pi}{n}$ tende para zero. Dessa forma, o fator

$$
\frac{\operatorname{sen}\left(\frac{\pi}{n}\right)}{\left(\frac{\pi}{n}\right)}
$$

tende para 1 , enquanto que o fator

$$
\frac{\operatorname{sen}\left(\frac{\pi}{n}\right)}{\cos \left(\frac{\pi}{n}\right)+1}
$$


tende para zero. Portanto,

$$
\lim _{n \rightarrow \infty} f_{M}=2 R .
$$

Para visualizar de maneira simples a convergência de $\mathrm{f}_{\mathrm{M}}$, basta lembrar que o Teorema Japonês é válido para qualquer tipo de triangularização, ou seja, garante que $\mathrm{f}_{\mathrm{M}}$ é uma constante. Dessa forma, podemos escolher uma triangularização onde todos os inraios sejam paralelos a um diâmetro do círculo. As figuras 22 e 23 ilustram os casos para $n=10$ e $n=20$. Repare que, à medida que o número de lados $\mathrm{n}$ do polígono aumenta, $\mathrm{f}_{\mathrm{M}}$ tende a ficar cada vez mais próxima do diâmetro do círculo.

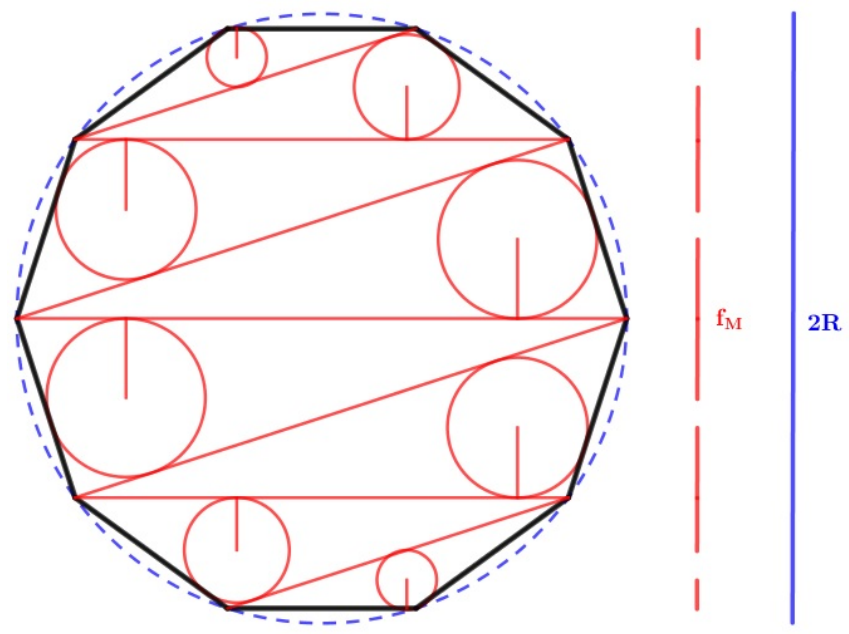

Figura 22: Função $\mathrm{f}_{\mathrm{M}}$ quando $\mathrm{n}=10$. 

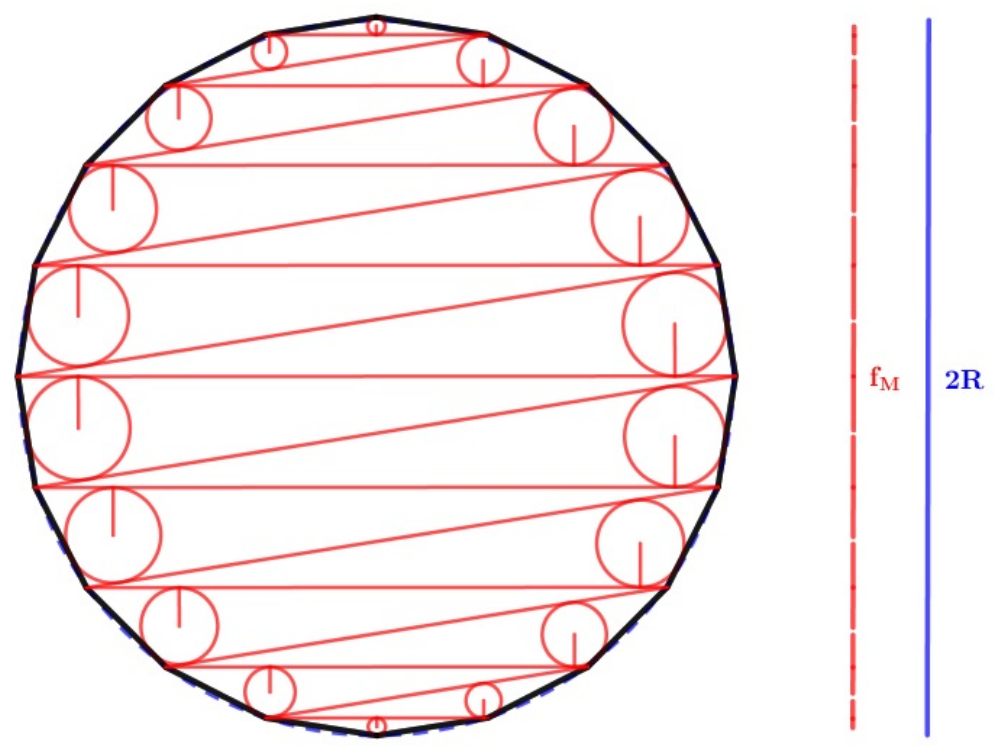

Figura 23: Função $f_{M}$ quando $n=20$.

\section{Agradecimentos}

Aos editores da revista PMO pela valiosa contribuição.

\section{Referências}

[1] Ahuja, M.; Uegaki, W.; Matsushita, K. "Japanese Theorem: A little known theorem with many proofs". Missouri Journal of Mathematical Sciences, v. 16, p. 72, 2004.

[2] Ahuja, M.; Uegaki, W.; Matsushita, K. In search of "The Japanese Theorem". Missouri Journal of Mathematical Sciences, v. 18, p. 87, 2006.

[3] Andreescu, T; Andrica, D. Complex Numbers from A to ... Z. Boston: Birkhäuser, 2006.

[4] Carvalho, P. C. P.; Morgado, A. C. Matemática Discreta. Coleção Profmat, $2^{\mathrm{a}}$ edição, Rio de Janeiro, SBM , 2015.

[5] Fujita, K. Zoku-Sinpeki-Sanpõ, vol 2, 1807.

[6] Fukagawa, H.; Pedoe, D. Japanese Temple Geometry Problems. The Charles Babbage Research Center, Winnipeg, Canadá, 1989.

[7] Greenstreet, W. "Japanese Mathematics". The Mathematical Gazette, v. 3, p. 268-270, 1906.

[8] Gusman, R. An old Japanese theorem. The Collection, nº 8, p. 3-7, 2003.

[9] Hawn, C. A Study of the Japanese Theorem. Masters Degree Thesis, South-east Missouri State University, 1996.

[10] Hayashi, T. Sur un Soi-Disant Theoreme Chinois. Mathesis, p. 257, 1906.

[11] Hosking, R. Sangaku: A Mathematical, Artistic, Religious, and Diagrammatic Examination. Doctoral Thesis, School of Mathematics and Statistics, University of Canterbury, 2016. 
[12] Lima, E. L. Curso de análise, v. 2. Coleção Projeto Euclides, 11ª edição, Rio de Janeiro, IMPA, 2018.

[13] Mackinnon, N. "Friends in Youth". The Mathematical Gazette, v. 77 p. 2-25, 1993.

[14] Mikami, Y. "A Chinese Theorem in Geometry". Archiv der Mathematik und Physik, v. 3, p. 308-310, 1905.

[15] Minculete, N.; Barbu, C.; Szollosy, G. "About the Japanese theorem", Crux Mathematicorum, v. 38, p. 188-193, 2012.

[16] Neto, A. C. M. Geometria. Coleção Profmat, 1ª edição, Rio de Janeiro, SBM , 2013.

[17] Richeson, D. The Japanese Theorem for Nonconvex Polygons - A Proof of the Generalized Japanese Theorem. MAA Publications, NW Washington D.C.:Mathematical Association of America, 2013. Disponível em: <https://scholar.dickinson.edu/faculty_publications/1405/>. Acesso em: 26 de setembro de 2021.

[18] Stewart, J. Cálculo, v. 2. 4ª edição, São Paulo, Pioneira Thomson Learning , 2001.

[19] Uegaki, W. "On the Origin and History of the Japanese Theorem". Journal of Mie University, 2001.

[20] Vieira Jr, I. F. Polígonos cíclicos e o teorema japonês. Dissertação de Mestrado, Universidade do Estado do Rio de Janeiro, PROFMAT, 2020.

[21] Yoshida, T. Zoku Shinpeki Sanpo Huroku Kai. Manuscrito sem data.

Italo Francisconi Vieira Júnior

Colégio Estadual Miguel Couto, Cabo Frio, RJ $<$ italofrancisconi.dt@gmail.com>

Fábio Silva de Souza Faculdade de Formação de Professores - UERJ, São Gonçalo, RJ $<$ fasouza08@gmail.com>

Recebido: 24/11/2020

Publicado: 11/11/2021 